\title{
The Different Stages of PRPD Pattern for Positive Point to Plane Corona Driven by a dc Voltage Containing a Ripple
}

Tobias Dezenzo

Technological University Dublin

Thomas Betz

Darmstadt University of Applied Sciences, Germany

Andreas Schwarzbacher

Technological University Dublin, andreas.schwarzbacher@tudublin.ie

Follow this and additional works at: https://arrow.tudublin.ie/ahfrcart

Part of the Electrical and Electronics Commons

\section{Recommended Citation}

Desenzo, T., Betz, T. \& Schwarzbacher, A. (2018). The Different Stages of PRPD Pattern for Positive Point to Plane Corona Driven by a dc Voltage Containing a Ripple. IEEE Transactions on Dielectrics and Electrical Insulation, vol. 25, no. 1. doi:10.1109/TDEI.2018.006670

This Article is brought to you for free and open access by the Antenna \& High Frequency Research Centre at ARROW@TU Dublin. It has been accepted for inclusion in Articles by an authorized administrator of ARROW@TU Dublin. For more information, please contact arrow.admin@tudublin.ie, aisling.coyne@tudublin.ie, gerard.connolly@tudublin.ie.

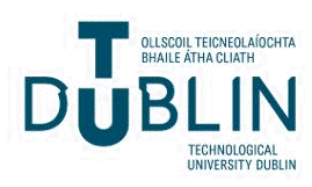




\title{
The Different Stages of PRPD Pattern for Positive Point to Plane Corona Driven by a dc Voltage Containing a Ripple
}

\author{
Tobias Dezenzo ${ }^{1,2}$, Thomas Betz ${ }^{1}$ and Andreas Schwarzbacher ${ }^{2}$ \\ ${ }^{1}$ Darmstadt University of Applied Sciences \\ Birkenweg 8 \\ 64295 Darmstadt, Germany \\ ${ }^{2}$ Dublin Institute of Technology \\ Kevin Street \\ Dublin 8, Ireland
}

\begin{abstract}
The different phase resolved partial discharge patterns, which can be recognized when a positive dc voltage containing a ripple due to a half-wave rectification is used are presented in this paper. Results are given from corona onset to the appearance of breakdown streamers. The outcomes are five different patterns, which represent the four stages of positive corona and their transition zones. Three of them are clearly identifiable with respect to patterns from other discharge sources that have already been reported for dc voltages containing a ripple. For an assessment of pattern formation discharge pulses as recorded at the test object are presented. Finally, the appearance of patterns is explained with support of the measured discharge pulses and the underlying physical processes.
\end{abstract}

Index Terms - partial discharges, ripple, HVDC, PRPD, corona, positive glow discharges, burst pulses, streamers, pattern.

\section{INTRODUCTION}

The growing demand for long range energy transportation has brought the topic of high voltage dc (HVDC) systems and their diagnostics back into the focus of much research activity. Of particular interest are partial discharge (PD) measurements and their evaluation, as PD is a commonly used indicator for weak spots in dielectrics and therefore in electrical insulation.

For alternating voltage a technique called phase resolved partial discharge (PRPD) representation is widely used. It relates the synchronously recorded basic quantities of PD measurement, discharge magnitude and their time of occurrence, to the phase angle of an ac signal. For this purpose it divides recorded PD data into sections with a length equal to the period of the applied alternating voltage, maps the time of occurrence onto an angle between 0 and 360 degrees and overlays all the sections to form one image. The advantage of this method is that every PD source e.g. corona or a void in a solid dielectric, forms a unique image (pattern). This grants the ability to distinguish between different types of defects.

However, in the area of dc diagnostics this method is not directly applicable as no phase relation is available. Therefore, other methods e.g. [1-4] have been proposed and are under development, which do not require a repeating sequence. All of them have in common that they require a higher level of expertise as well as post-processing effort compared to the ac PRPD method.

To overcome these problems a method, which enables the use of PRPD for dc PD purposes by superimposing a repeating sequence has been proposed $[5,6]$. The repeating sequence is required to synchronize the PD detection and results have already been reported for different types of discharge sources [6-8]. In this paper the method is applied to a point to plane arrangement being driven by a positive dc voltage. The required repeating sequence is automatically present due to the fact that a half-wave rectification of a $50 \mathrm{~Hz}$ ac signal is used to obtain the dc voltage. The resulting patterns from discharge onset to the appearance of breakdown streamers are given and explained by means of their physical processes.

\section{CONFIGURATION OF THE TEST SETUP AND REPRESENTATION OF THE DATA}

\subsection{MEASUREMENT SETUP}

A test circuit according to Figure 1 has been used. The supply of the test setup is composed of a low voltage adjustable transformer in combination with a fixed ratio high voltage transformer. The test setup can provide up to $100 \mathrm{kV}$ and is PD free up to $80 \mathrm{kV}$. One Omicron MPD 600 
is connected via a quadripole to the coupling capacitor $C_{k 1}$ to synchronize the PD detection. To obtain a dc voltage a half wave rectification of the ac signal is performed. Two diodes are connected in series to reduce the individual voltage drop per diode. An inductance $L$ is used to separate the test object and the PD detection circuit from the grid.

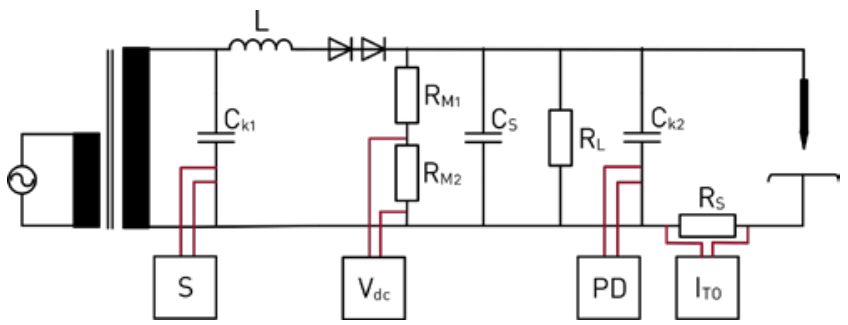

Figure 1. Equivalent circuit of the measuring setup for a point-to-plane configuration including the devices for synchronization $S$, voltage measurement on the dc side $V_{d c}$, partial discharge decoupling $P D$ and discharge current measurement ITO.

A voltage divider consisting of a resistor $R_{M 1}$ being equal to $1 \mathrm{G} \Omega$ and $R_{M 2}$ being the $1 \mathrm{M} \Omega$ input impedance of a HBM GN110 probe is used to measure the voltage on the dc side of the circuit. The smoothing capacitor $C_{S}$ has a capacity of $10 \mathrm{nF}$. The purpose of the load resistor $R_{L}$ is to adjust the time constant of the dc circuit and therefore the ripple on the dc voltage. If a very smooth voltage is desired $R_{L}$ can be replaced by an additional $10 \mathrm{nF}$ capacitor. This has been done to perform reference measurements. In case that the voltage contains a ripple the load resistor has been set to $10 \mathrm{M} \Omega$. The result is a ripple of about $5.5 \%$, which is almost twice as large as the recommended maximum ripple in IEC $60060-1$ of $3 \%$. This comparatively large ripple has been used to delineate the boarders between different types of discharges more clearly. Even though, this ripple seems large it still falls within the ripple range for HVDC applications of $2 \%$ to $10 \%$ as reported in [9].

In general it can be shown that even a very small ripple will have a visible effect by means of a pattern in the case of positive corona. Small in this context means that the ripple is well below the 3 \% recommendation of IEC 60060-1. This is indicated in Figure 2, where a $Q(\phi)$-diagram is shown for a ripple of $0.1 \%$. Additional support for this

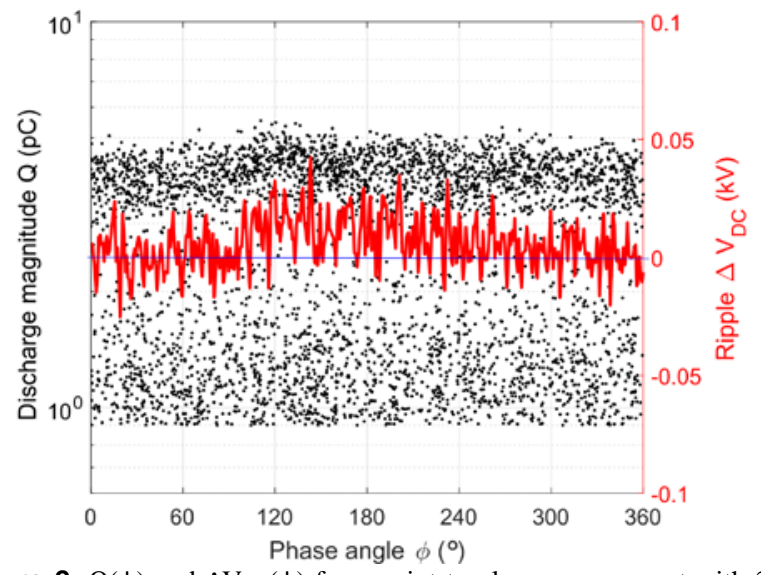

Figure 2. $\mathrm{Q}(\phi)$ and $\Delta \mathrm{V}_{\mathrm{DC}}(\phi)$ for a point to plane arrangement with 30 mm electrode interspacing at $19.3 \mathrm{kV}$ dc with a ripple of $0.1 \%$. postulation is given by Figure 3, where the current at the test object $I_{T O}$ and its dependence upon the applied voltage $V$ in the glow regime is shown.

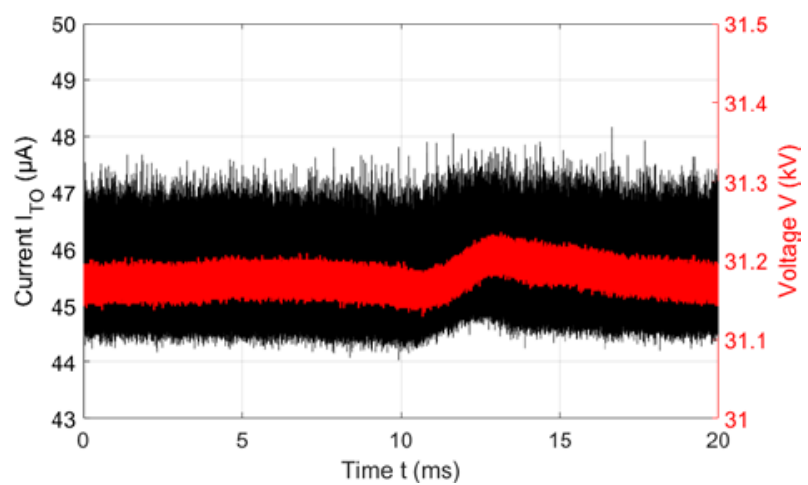

Figure 3. $\mathrm{ITO}_{\mathrm{TO}}(\mathrm{t})$ and $\mathrm{V}(\mathrm{t})$ for a point to plane arrangement with $30 \mathrm{~mm}$ electrode interspacing at $31.2 \mathrm{kV}$ dc with a ripple of $0.1 \%$.

A second Omicron MPD 600 is connected via a quadripole to the coupling capacitor $C_{k 2}$ to measure the partial discharges coming in from the test object. The capacity of the coupling capacitor $C_{k 2}$ is $1200 \mathrm{pF}$. A $1 \mathrm{k} \Omega$ shunt resistor $R_{S}$ is connected in series to the test object to measure the current $I_{T O}$ through the test sample. The current recording is performed using a HBM GN110 probe in combination with a HBM Gen7t. This transient recorder has a resolution of 14 bit and provides $100 \mathrm{MS} / \mathrm{s}$.

The test object (Figure 4) is a point to plane arrangement, with a point radius $r_{N}$ of $0.5 \mathrm{~mm}$ and a rogowski-shaped plate of radius $r_{P}$ being equal to $60 \mathrm{~mm}$. The interspacing $s$ has been set to $30 \mathrm{~mm}$. The environmental conditions were determined and showed a temperature of $20.5^{\circ} \mathrm{C}$, a relative humidity of $30 \%$ and an atmospheric pressure of $1001 \mathrm{hPa}$. The measurements have been performed in accordance to IEC 60270. The filter properties of the bandpass filter have been set to a lower limit frequency $f_{1}$ of $100 \mathrm{kHz}$ and an upper limit frequency $f_{2}$ of $400 \mathrm{kHz}$. The PD detection device is set to continuously record PD activity over $120 \mathrm{~s}$.

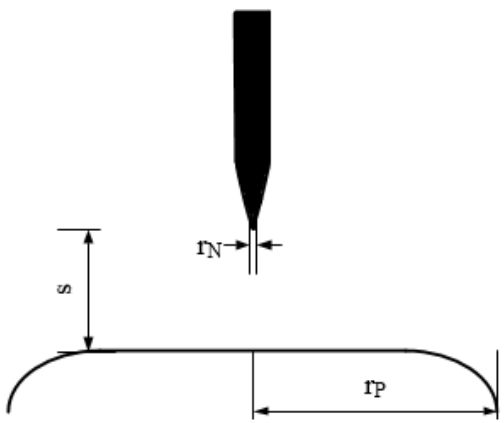

Figure 4. Scheme of the point to plane test object.

\subsection{DATA REPRESENTATION}

In this paper different graphs with various dependencies are used to present the gathered data. These different representations are:

1) A $Q(\phi)$-diagram, displaying the discharge magnitude $Q$ in relation to the phase angle $\phi$ of the applied 
voltage. On a secondary y-axis the ripple $\Delta V_{D C}$ as function of the phase angle $\phi$ is presented. This graph type shows the resulting PRPD patterns.

2) $I_{T O}(t)$-diagram, which shows the discharge current at the test object $I_{T O}$ over at least a $20 \mathrm{~ms}$ time sequence. On another y-axis the applied voltage $V$ is given. The former are referred to as part (a) of such a figure, while a zoomed version of a pulse is referred to as part (b).

3) $Q(t)$-diagram, showing the discharge magnitude $Q$ as a function of time $t$.

4) $\Delta Q(\Delta t)$-diagram, which shows the magnitude difference $\Delta Q$ (equation (1)) between a discharge $Q(t)$ and its successor $Q\left(t_{s}\right)$ as a function of their time difference $\Delta t$ (equation (2)). It is distinguished between a positive and negative difference i.e. an increasing or decreasing magnitude.

$$
\begin{aligned}
& \Delta Q=Q\left(t_{s}\right)-Q(t) \\
& \Delta t=t_{s}-t
\end{aligned}
$$

All voltages are presented as mean values and are therefore given in terms of the dc component of the applied voltage only. To obtain the maximum or the minimum one has to add or subtract $5.5 \%$ of the mean value.

\section{RESULTS AND DISCUSSION}

The first stage of positive corona discharge activity can be observed at $18.1 \mathrm{kV}$ (Figure 5). A concentration of discharges with magnitudes smaller than $10 \mathrm{pC}$ occurring in the vicinity of the maximum of the applied voltage is recognizable. This recording has been made in the high sensitivity mode of the PD measuring device, which enables the use of the highest PD input gain. The high gain leads to a background noise that is smaller than $0.6 \mathrm{pC}$ and has been excluded from the figure.

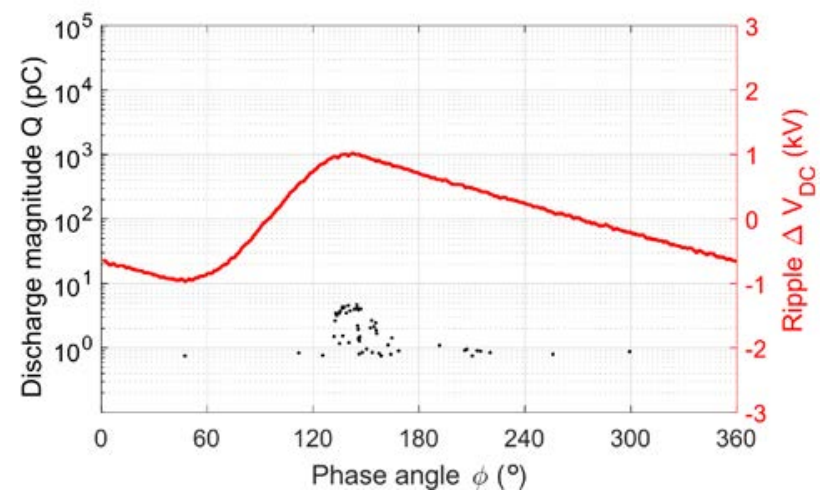

Figure 5. $\mathrm{Q}(\phi)$ and $\Delta \mathrm{V}_{\mathrm{DC}}(\phi)$ for a point to plane arrangement with 30 mm electrode interspacing at $18.1 \mathrm{kV}$ dc with a ripple of $5.5 \%$.

The concentration of the discharges in the maximum can be explained with the inception voltage, which has to be reached to enforce a self-sustained discharge. It is reached in the part of the cycle where PD activity is detected and is not reached during the rest of the cycle. Figure 6 (a) shows a 20 ms recording of the discharge current, which has been measured in series with the test object. A pulse in the

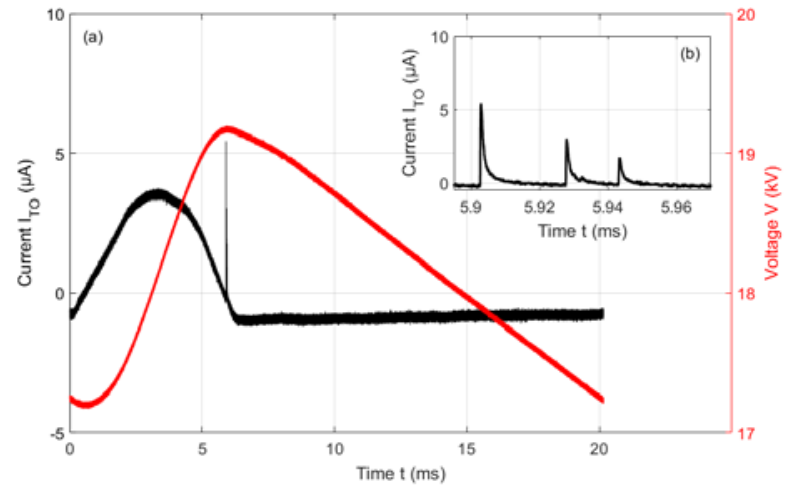

Figure 6. $\mathrm{ITO}_{\mathrm{TO}}(\mathrm{t})$ and $\mathrm{V}(\mathrm{t})$ for a point to plane arrangement with $30 \mathrm{~mm}$ electrode interspacing at $18.1 \mathrm{kV}$ dc with a ripple of $5.5 \%$ (a) and a zoomed version (b).

maximum of the voltage as well as a sinusoidal half-wave can be seen. As a sinusoidal half-wave corresponds to the first derivative of a negative cosine function, it indicates a capacitive behaviour. The sinusoidal waveform in the current is therefore caused by the ripple of the applied voltage and is not related to discharge activity.

If the discharge current pulse in the maximum of the voltage is closer examined (Figure 6 (b)) three pulses with decreasing amplitude and decreasing time interspacing are visible. A similar pulse series is depicted in Figure 7, which shows a part of a $Q(t)$ representation of the data being contained in Figure 5. The strong correlation between the actual discharge pulses and the recorded discharge magnitudes gives rise to the postulation that the $Q(\phi)$ representation in Figure 5 results from pulses as per Figure 6 . These pulses are comparable to the pulses reported by Miyoshi and Hosokawa [10], who used a similar geometric arrangement. They are referred to as burst pulses in the literature e.g. [11]. This type of discharge spreads along the anode surface [12] and is then choked off by the developed positive space charge [13]. Free electrons then move to another part of the anode [11], where a new discharge can start. This sequence will continue until the ionization is completely suppressed by the positive ions [13].

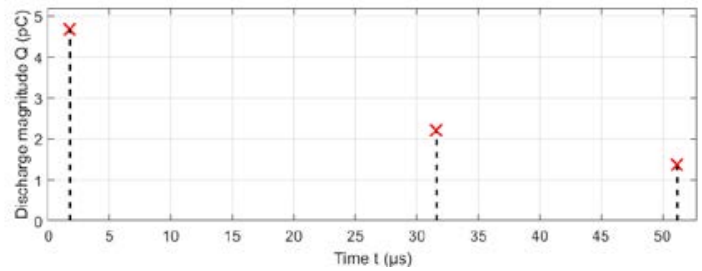

Figure 7. Q(t) for a point to plane arrangement with $30 \mathrm{~mm}$ electrode interspacing at $18.1 \mathrm{kV}$ dc with a ripple of $5.5 \%$.

The first occurring pattern is therefore formed due to burst pulse activity.

It has to be noted that this pattern is rather similar to a pattern for internal PD observed during a study [7] on PRPD for negative dc voltages containing a ripple. No data for a positive voltage has been published so far but a concentration of discharges in the vicinity of the maximum of the applied voltage is likely. A differentiation might therefore only be possible by the number of discharges, which show a general tendency of being less frequent for 
internal PD then for positive corona. This holds true if the ripple on the dc voltage is small enough to not cause a discharge every $50 \mathrm{~Hz}$ cycle.

The burst pulse pattern is therefore not recommended as source identification.

If the voltage is slightly increased the discharge magnitude in the vicinity of the maximum of the voltage shows two levels. One is in the $\mathrm{nC}$ range and the other one is in the $\mathrm{pC}$ range. Discharges occurring at values adjacent to the maximum remain in the $\mathrm{pC}$ area (Figure 8). The gain of the PD detection system had to be changed to protect the measuring device which results in a higher background noise that is now up to $2.5 \mathrm{pC}$ and has been excluded from the graphs.

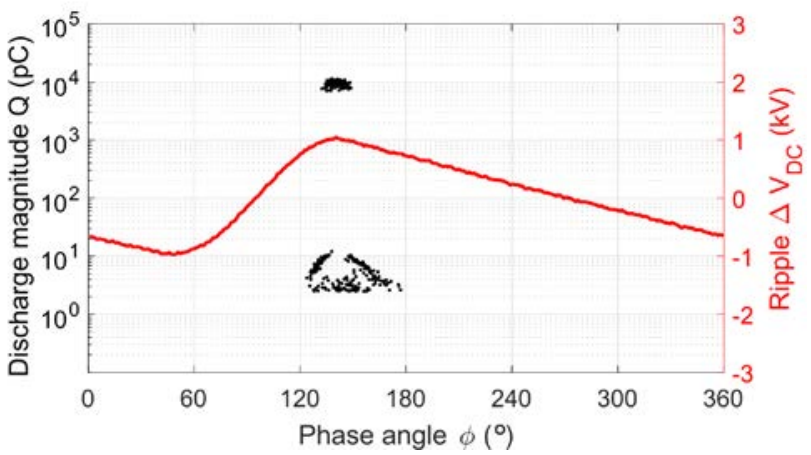

Figure 8. $\mathrm{Q}(\phi)$ and $\Delta \mathrm{VDC}(\phi)$ for a point to plane arrangement with 30 mm electrode interspacing at $18.3 \mathrm{kV}$ dc with a ripple of $5.5 \%$.

To more accurately assess the sudden change in discharge magnitude, current pulses as recorded at the test object are evaluated. Burst pulse activity as indicated in Figure 6 is still present, but a new pulse type (Figure 9) can be observed.

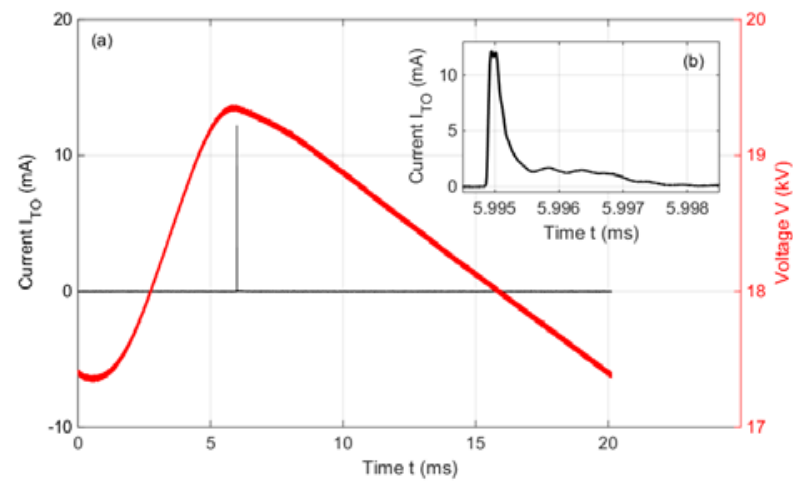

Figure 9. $\mathrm{ITO}_{\mathrm{TO}}(\mathrm{t})$ and $\mathrm{V}(\mathrm{t})$ for a point to plane arrangement with $30 \mathrm{~mm}$ electrode interspacing at $18.3 \mathrm{kV}$ dc with a ripple of $5.5 \%$ (a) and a zoomed version (b).

The pulse does again occur in the maximum of the voltage but has a peak value that is at least a thousand times larger than the peak value of the pulses as per Figure 6. This type of pulse is referred to as a pre-onset streamer [14] or onset streamer $[11,15]$. On the contrary to the burst pulse this type of discharge does evolve radially into the gap. It is primary driven by photoionization $[14,16]$ and ceases due to positive ion formation near the anode [11].

As the differences of the peak values of the pulses are comparable to the differences in discharge magnitudes, it must be concluded that it are these streamer pulses, which are responsible for the detected discharges in the $\mathrm{nC}$ range.

For a more precise understanding of the underlying processes of pattern formation it is also of interest whether or not the two different recorded pulses are in a specific relation to each other or not. Therefore, the recorded data has been transferred into a $\Delta Q(\Delta t)$ representation, which shows the dependency of the discharge magnitude on the time interspacing between pulses. The $\Delta Q(\Delta t)$ graph shown in Figure 10 emphasizes that the general time interspacing tendency is towards times that are larger than one $20 \mathrm{~ms}$ period. The time required between streamers has been estimated to be in the range of $1 \mathrm{~ms}$ [14]. In this study however times between streamers have been estimated to be in the order of seconds close to their onset. Nevertheless, as times between discharges appear in discrete steps of about $20 \mathrm{~ms}$ a mainly inception voltage triggered behavior is indicated. A relation between streamers and burst pulses can be seen in the micro second area of the graph. Here a large pulse (streamer) is almost always followed by a small pulse $(\Delta Q<0 ; 20 \mu \mathrm{s}$ to $30 \mu \mathrm{s})$. Furthermore, small pulses might also be followed by small pulses but with a time interspacing that is smaller than $10 \mu \mathrm{s}$. The latter finding corresponds to a burst pulse behavior. Therefore, the wellknown streamer is followed by a burst pulse arithmetic can be observed or both discharge types can appear individually $(\Delta t>20 \mathrm{~ms})$.

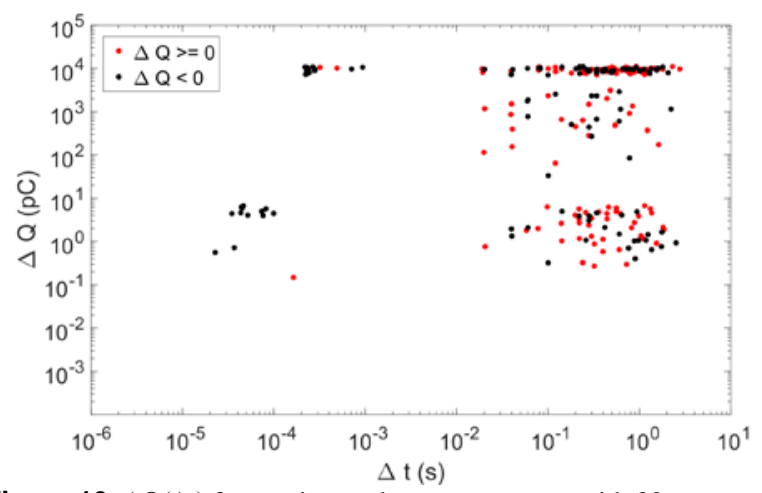

Figure 10. $\Delta \mathrm{Q}(\Delta \mathrm{t})$ for a point to plane arrangement with $30 \mathrm{~mm}$ electrode interspacing at $18.3 \mathrm{kV}$ dc with a ripple of $5.5 \%$.

An enhancement of the voltage to $18.7 \mathrm{kV}$ provides a $\Delta Q(\Delta t)$ graph as per Figure 11. From this graph it is evident that the "streamer being followed by a burst pulse"-scheme $(\Delta Q<0)$ becomes more dominant, as well as a strongly enhanced discharge activity with multiple streamer pulses appearing during one period (see $\Delta Q>0$ in the $100 \mu s \Delta t$ area).

The occurrence of more discharges can on the one hand be argued with the inception voltage, which is reached for a longer time with respect to the period of the applied voltage and on the other hand with an enhanced ion mobility due to the higher field strength.

This explanation is supported by Figure 12, which indicates that the discharge magnitude as well as the discharge frequency exhibit a dependency upon the voltage height. The discharge magnitude is the highest in the 


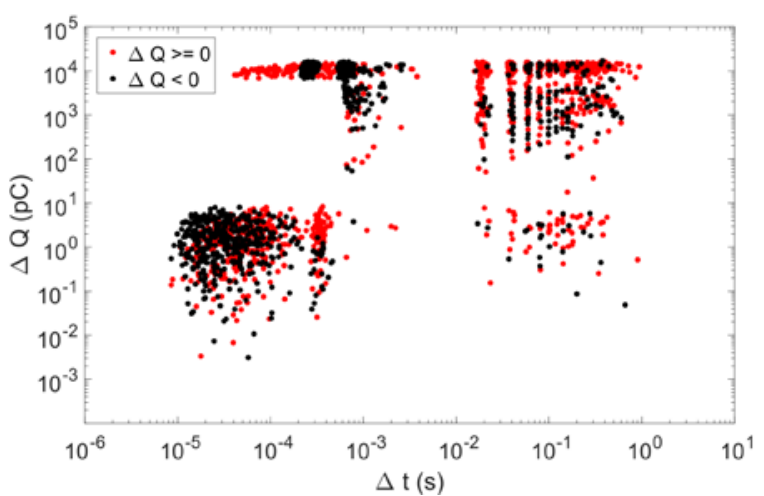

Figure 11. $\Delta \mathrm{Q}(\Delta \mathrm{t})$ for a point to plane arrangement with $30 \mathrm{~mm}$ electrode interspacing at $18.7 \mathrm{kV}$ dc with a ripple of $5.5 \%$.

maximum of the applied voltage and decreases in the same matter as the voltage waveform. This behavior combined with the two different levels of discharge magnitude can be used to identify positive corona as the PD source.

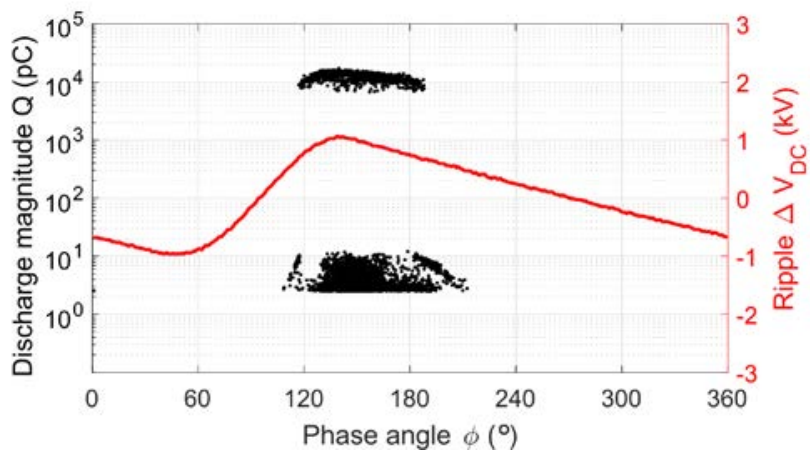

Figure 12. $\mathrm{Q}(\phi)$ and $\Delta \mathrm{VDC}(\phi)$ for a point to plane arrangement with 30 $\mathrm{mm}$ electrode interspacing at $18.7 \mathrm{kV}$ dc with a ripple of $5.5 \%$.

A sequence, where a streamer is followed by a burst pulse is depicted in Figure 13. The large overshoot in part (b) of Figure 13 is caused by the settings of the measuring device. These have been set to give a good resolution of the streamer (Figure 13 (a)) and of the burst pulse (Figure 13 (b)).
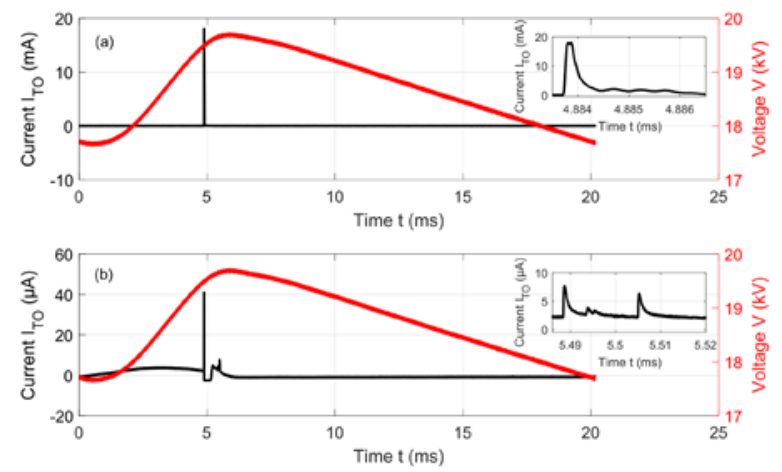

Figure 13. $\mathrm{I}_{\mathrm{TO}}(\mathrm{t})$ and $\mathrm{V}(\mathrm{t})$ for a point to plane arrangement with $30 \mathrm{~mm}$ electrode interspacing at $18.7 \mathrm{kV}$ dc with a ripple of $5.5 \%$. Measuring resolution set to accurately resolve the streamer pulse (a) and the burst pulse series (b).

If the voltage is further increased the inception voltage for burst pulses and onset streamers is reached over a wider range of the phase angle (Figure 14). This is evident by the forth growing of the low magnitude and the high magnitude discharge regions to higher and lower phase angles with respect to the position of the maximum of the applied voltage.

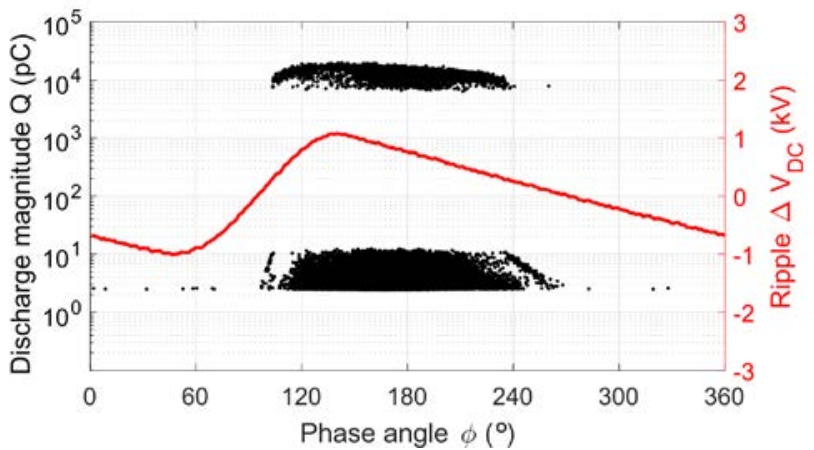

Figure 14. $\mathrm{Q}(\phi)$ and $\Delta \mathrm{V}_{\mathrm{DC}}(\phi)$ for a point to plane arrangement with 30 mm electrode interspacing at $19.2 \mathrm{kV}$ dc with a ripple of $5.5 \%$.

At $20.0 \mathrm{kV}$ the pattern is still forth growing with respect to the phase angle but begins to shrink starting from the voltages maximum (Figure 15). This marks the beginning transition from the streamer / burst pulse region to the glow regime and can be used as another identification property of positive corona. Additionally a $60 \mathrm{~ms}$ time sequence of pulses as recorded at the test object is shown in Figure 16.

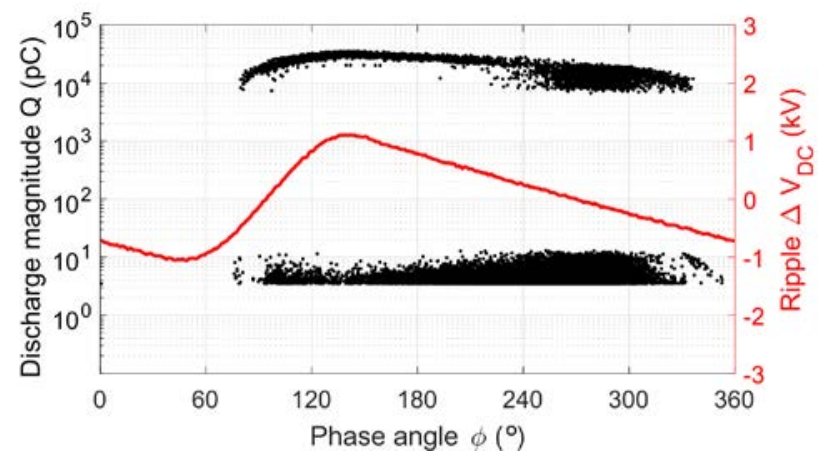

Figure 15. $\mathrm{Q}(\phi)$ and $\Delta \mathrm{V}_{\mathrm{DC}}(\phi)$ for a point to plane arrangement with 30 $\mathrm{mm}$ electrode interspacing at $20.0 \mathrm{kV}$ dc with a ripple of $5.5 \%$.

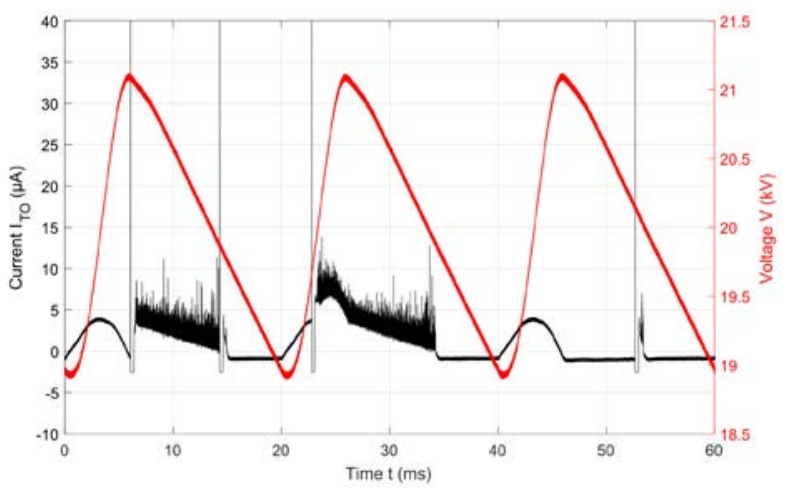

Figure 16. Iто(t) and $\mathrm{V}(\mathrm{t})$ for a point to plane arrangement with $30 \mathrm{~mm}$ electrode interspacing at $20.0 \mathrm{kV}$ dc with a ripple of $5.5 \%$.

It is evident that streamers are still followed by burst pulses. The latter can be of short or long duration, mainly depending upon the point of streamer occurrence in relation to the applied voltage. Even though, the constant current component is highest in the maximum of the voltage the amplitude of the single pulses appearing in a burst is lower 
close to the maximum than in lower voltage regions. As the constant current component is cut off due to the capacitive PD decoupling the resulting charge delivered per burst pulse will be smallest in the maximum of the voltage. This explains the shrinking of the pattern. The reduced measured discharge activity can be explained in the same way. In general the discharge frequency of burst pulses is proportional to the positive ion mobility [17, 18] and therefore to the height of the applied voltage. This could also be observed in this study. If Figure 16 is examined from $25 \mathrm{~ms}$ to $35 \mathrm{~ms}$ an anti-proportional relation between voltage and individual pulse height i.e. without the dc component, is visible in the glow onset region. This is in contradiction to simulative studies [17, 19] but in fair agreement with experimental studies [20]. At higher voltages the amplitudes are nearly constant (Figure 19, Figure 20).

The pulses close to the maximum of the voltage show pulse amplitudes (without dc component), which are mostly smaller than $2 \mu \mathrm{A}$. A small fraction of pulses with amplitudes of up to $5 \mu \mathrm{A}$ is visible. It was shown earlier (e.g. Figure 5 and Figure 6) that the pulse amplitudes give a first estimate of the discharge magnitude range in terms of pC. If a $2 \mu \mathrm{A}$ pulse would yield a 2 pC pulse then this pulse would not be visible as a data point in the $Q(\phi)$-diagram as the level of background noise is at $2.5 \mathrm{pC}$. Therefore, it can be concluded that the discharge activity is enhanced but due to the reduced amplitudes they are simply hidden in the background noise.

If the voltage is increased to $21.4 \mathrm{kV}$ the discharges seem to cease starting from the maximum of the voltage as indicated in Figure 17. A corresponding time series is shown in Figure 18. A streamer in the vicinity of the minimum of the applied voltage is visible, which is followed and preceded by a series that is similar to a never ending burst pulse. It is also evident that the current is not returning to zero at any given point in the series. This appearance is known as the glow regime of positive corona [21].

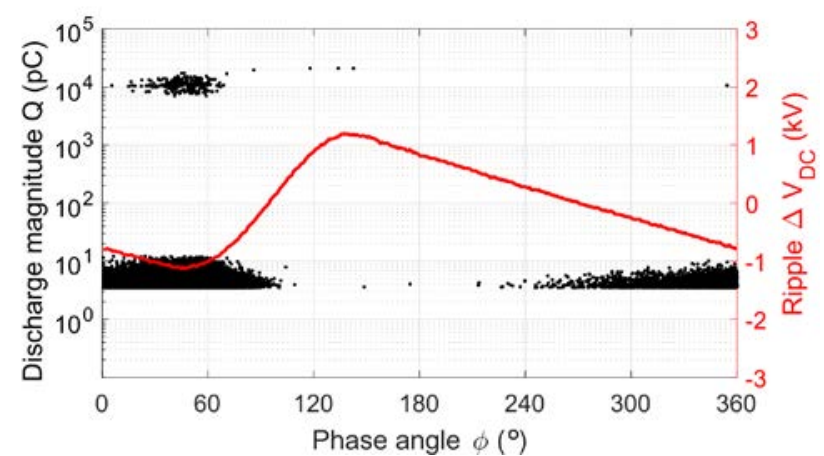

Figure 17. $\mathrm{Q}(\phi)$ and $\Delta \mathrm{VDC}(\phi)$ for a point to plane arrangement with 30 mm electrode interspacing at $21.4 \mathrm{kV}$ dc with a ripple of $5.5 \%$

Figure 18 furthermore shows that the discharge activity does not stop but leads to charge magnitudes, which are below the threshold value of the background noise. If the voltage is further increased then the pattern vanishes completely. Therefore, it is not possible to show an

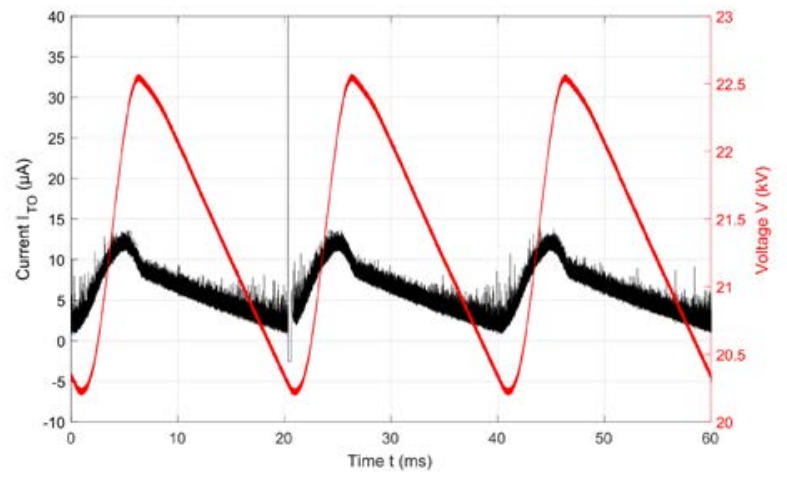

Figure 18. $\mathrm{I}_{\mathrm{TO}}(\mathrm{t})$ and $\mathrm{V}(\mathrm{t})$ for a point to plane arrangement with $30 \mathrm{~mm}$ electrode interspacing at $21.4 \mathrm{kV}$ dc with a ripple of $5.5 \%$

evolvement of the PRPD pattern in the glow regime. Nevertheless, it is still possible to make an estimate of the change of pattern based on the measured current pulses at the test object.

A visual examination of the pulsating nature of the glow regime reveals sinusoidal like and saw-tooth shaped pulses (Figure 19(b) and Figure 20(b)).
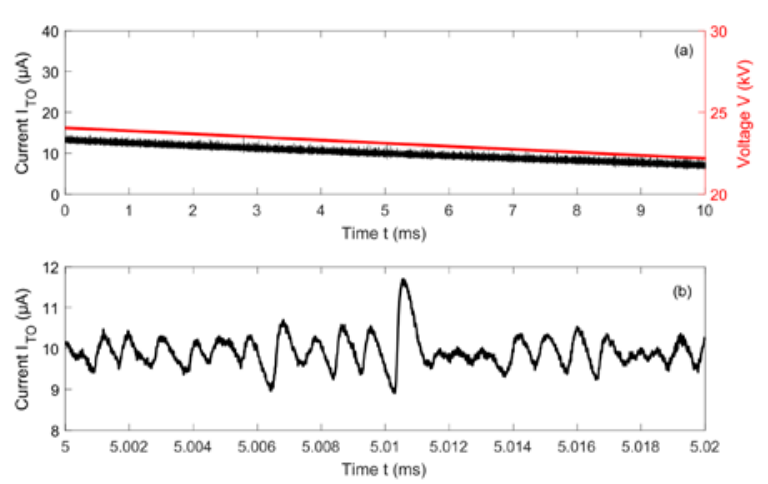

Figure 19. ITO $(\mathrm{t})$ and $\mathrm{V}(\mathrm{t})$ for a point to plane arrangement with $30 \mathrm{~mm}$ electrode interspacing at $23.0 \mathrm{kV}$ dc with a ripple of $5.5 \%$ (a) and a zoomed version (b).
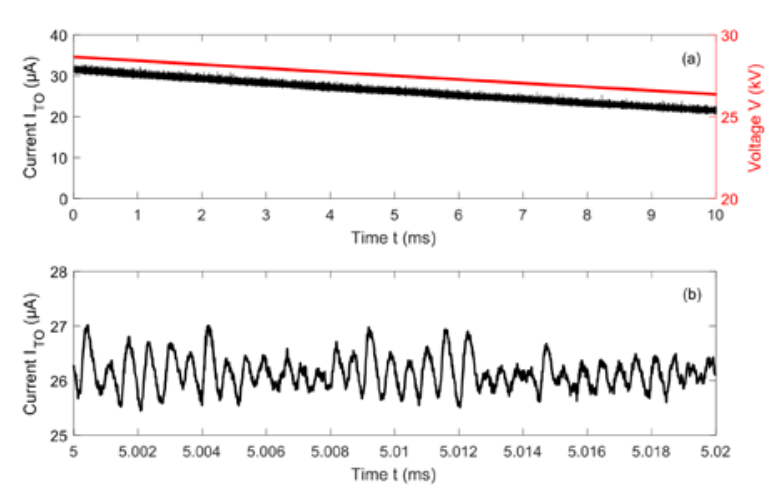

Figure 20. $\mathrm{I}_{\mathrm{TO}}(\mathrm{t})$ and $\mathrm{V}(\mathrm{t})$ for a point to plane arrangement with $30 \mathrm{~mm}$ electrode interspacing at $29.5 \mathrm{kV}$ dc with a ripple of $5.5 \%$ (a) and a zoomed version (b).

If the two previous figures are compared, then a large difference in discharge frequency is visible, while the amplitudes remain in the same region. Therefore, a resulting pattern will scatter around a certain average discharge magnitude, where no correlation to the waveform of the applied voltage might be found if the ripple is small compared to the voltage band where the glow discharge 
region is active. In case that the ripple is higher, then the pattern will show an inverse proportionality to the waveform of the applied voltage, leading to higher discharge magnitudes in the minimum, which is comparable to Figure 17. Even if a small ripple is used a differentiation might be possible based on the number of discharges per second as these will show a proportionality to the curve of the voltage.

For the purpose of discharge frequency estimation a DFT with a Hann window for the time series in Figure 19 (a) and Figure 20 (a) has been performed. The series has a duration of $10 \mathrm{~ms}$ and corresponds to discharges, which occurred in the linear part of the voltage decay. The results show a discharge frequency, which increases from about $1 \mathrm{MHz}$ to approximately $2 \mathrm{MHz}$ over a voltage range from about 22.5 $\mathrm{kV}$ to $28 \mathrm{kV}$ (Figure 21). The voltage values have been taken from Figure 19 (a) and Figure 20 (a), respectively.

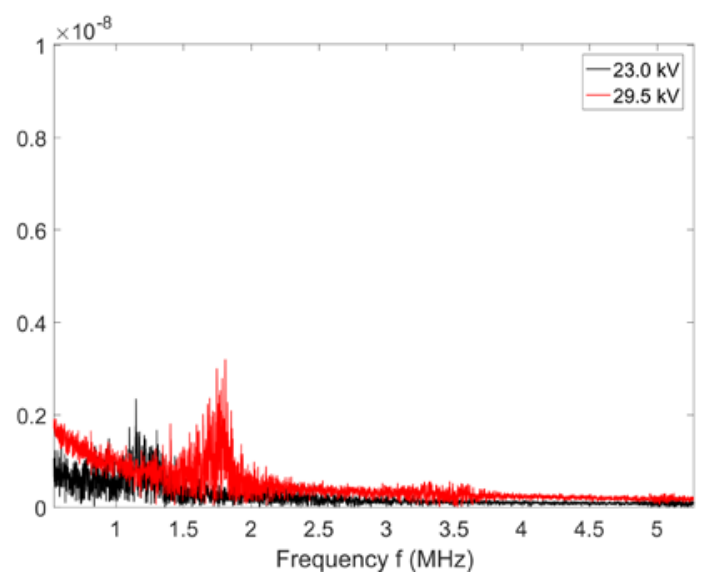

Figure 21. Frequency spectrum corresponding to the time series in Figure 19 (a) and Figure 20 (a).

The final pattern that has been recorded shows the reappearance of discharges out of the glow regime, as shown in Figure 22. The discharges occur with time interspacing of minutes and are of very large amplitudes. The resulting pattern is therefore not recommended for identification purposes. In literature e.g. [11] these are referred to as breakdown streamers. A corresponding pulse is shown in Figure 23.

The voltage has not been further enhanced to not provoke a breakdown that might potentially damage the measuring devices.

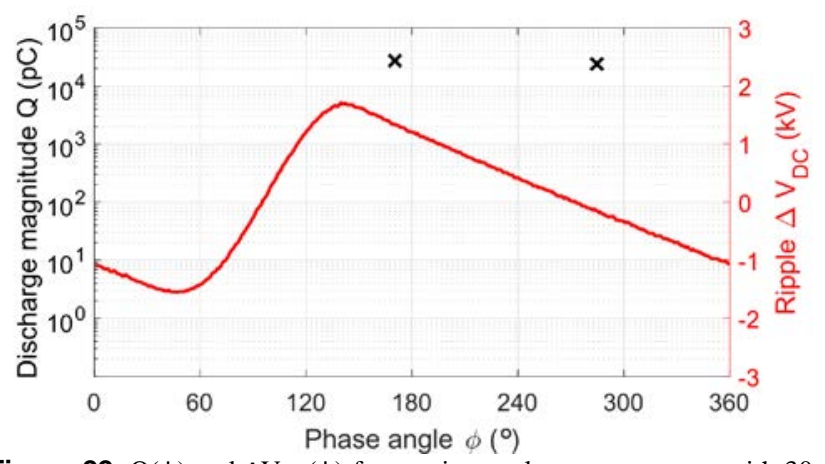

Figure 22. $\mathrm{Q}(\phi)$ and $\Delta \mathrm{V}_{\mathrm{DC}}(\phi)$ for a point to plane arrangement with 30 $\mathrm{mm}$ electrode interspacing at $29.5 \mathrm{kV}$ dc with a ripple of $5.5 \%$.

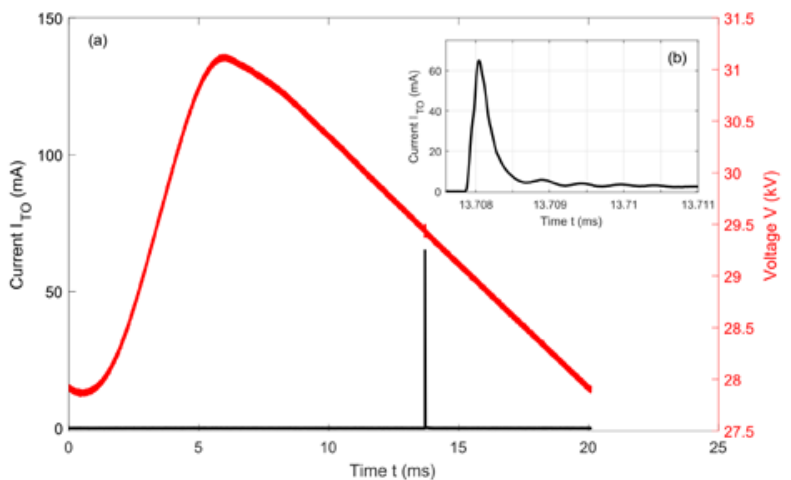

Figure 23. $\mathrm{I}_{\mathrm{TO}}(\mathrm{t})$ and $\mathrm{V}(\mathrm{t})$ for a point to plane arrangement with $30 \mathrm{~mm}$ electrode interspacing at $29.5 \mathrm{kV}$ dc with a ripple of $5.5 \%$ (a) and a zoomed version (b).

\section{CONCLUSION}

In this paper a point to plane arrangement with $30 \mathrm{~mm}$ electrode interspacing has been examined with respect to PRPD pattern formation. The novel feature is that even though a positive dc voltage is used the PRPD technique is applicable due to the present voltage ripple. The pattern evolution is presented from corona onset until the formation of breakdown streamers. Five different forms can be observed and are described as follows:

1.) If only burst pulses are present near onset a concentration of discharges in the maximum of the applied voltage is visible. Their magnitudes lay in the small pC area.

2.) An occurrence of streamers combined with burst pulses leads to two different magnitude levels. One level is in the $\mathrm{pC}$ range and the other in the $\mathrm{nC}$ range. The discharge magnitude in both levels follows the waveform of the applied voltage.

3.) In the beginning transition to the glow discharge the pattern begins to shrink starting from the maximum of the applied voltage.

4.) In regions where the glow inception voltage is reached the discharge magnitude will scatter around an average value. The discharge activity will follow the curve of the applied voltage. Depending upon the setting of the measuring system the pattern might completely disappear.

5.) Close to breakdown pulses occur with very large discharge magnitudes and long time interspacing.

As the presented patterns were related to the discharge currents occurring at the test object their cause of formation has been clearly explained. The presented patterns can for the first time be used to identify positive corona as the discharge source and furthermore judge the criticality based on the observed discharge type. This study has therefore contributed to the knowledge base of PRPD in general and especially if it is applied to dc measurements. 


\section{REFERENCES}

[1] P. Morshuis and J. J. Smit, "Partial discharges at dc voltage: their mechanism, detection and analysis," Dielectrics and Electrical Insulation, IEEE Transactions on, vol. 12, no. 2, pp. 328-340, 2005.

[2] A. Pirker and U. Schichler, "Partial discharges at DC voltage measurement and pattern recognition,” in 2016 International Conference on Condition Monitoring and Diagnosis (CMD), 2016, pp. 287-290.

[3] A. Cavallini, G. Montanari, M. Tozzi, and X. Chen, "Diagnostic of HVDC systems using partial discharges,” IEEE Trans. Dielect. Electr. Insul, vol. 18, no. 1, pp. 275-284, 2011.

[4] S. Wenrong, L. Junhao, Y. Peng, and L. Yanming, "Digital detection, grouping and classification of partial discharge signals at dc voltage," Dielectrics and Electrical Insulation, IEEE Transactions on, vol. 15, no. 6, pp. 1663-1674, 2008.

[5] T. Betz, T. Dezenzo, D. Sunaryadi, and F. Radler, "Teilentladungsdetektion und fehlererkennung in dc-systemen: neue ansätze,” in ETG-Fachbericht, vol. 144, Diagnostik elektrischer Betriebsmittel 2014: Beiträge der 6. ETGFachtagung 25. - 26. November 2014 in Berlin, Berlin: VDEVerlag, 2014.

[6] T. Dezenzo, T. Betz, and A. T. Schwarzbacher, "A new method for the evaluation and recognition of corona under direct voltage stress,” in Signals and Systems Conference (ISSC), 2015 26th Irish, 2015, pp. 1-6.

[7] T. Dezenzo, T. Betz, and A. Schwarzbacher, "Transfer and evaluation of the ac PRPD representation for internal PD at dc voltage," in 2016 IEEE International Conference on Dielectrics (ICD), 2016, pp. 528-531.

[8] T. Dezenzo, T. Betz, and A. T. Schwarzbacher, "The different stages of PRPD pattern for negative point to plane corona driven by a dc voltage containing a ripple,” IEEE Trans. Dielect. Electr. Insul, vol. 24, no. 1, pp. 47-53, 2017.

[9] CIGRÉ Working Group B4. 37, "VSC Transmission,” vol. 269, 2005.

[10] Y. Miyoshi and T. Hosokawa, "The formation of a positive corona in air," Journal of Physics D: Applied Physics, vol. 6, no. 6, p. 730, 1973.

[11] N. G. Trinh, "Partial discharge XIX: discharge in air part I: physical mechanisms," IEEE Electrical Insulation Magazine, vol. 11, no. 2, pp. 23-29, 1995.

[12] G. W. Trichel, "The mechanism of the positive point-to-plane corona in air at atmospheric pressure,” Phys. Rev, vol. 55, no. 4, pp. 382-390, 1939.

[13] L. B. Loeb, Electrical coronas, their basic physical mechanisms. Berkeley: Univ. of California Press, 1965.

[14] A. F. Kip, "Onset studies of positive point-to-plane corona in air at atmospheric pressure," Phys. Rev, vol. 55, no. 6, pp. 549-556, 1939.

[15] T. N. Giao and J. B. Jordan, "Modes of corona discharges in air," IEEE Transactions on Power Apparatus and Systems, vol. PAS87, no. 5, pp. 1207-1215, 1968.

[16] M. R. Amin, "Fast time analysis of intermittent point-to-plane corona in air. II. the positive pre-onset streamer corona," Journal of Applied Physics, vol. 25, no. 3, pp. 358-363, 1954.

[17] R. Morrow, "The theory of positive glow corona," Journal of Physics D: Applied Physics, vol. 30, no. 22, p. 3099, 1997.

[18] L. Liu and M. Becerra, "On the transition from stable positive glow corona to streamers," Journal of Physics D: Applied Physics, vol. 49, no. 22, p. 225202, 2016.

[19] L. Liu and M. Becerra, "An efficient model to simulate stable glow corona discharges and their transition into streamers," Journal of Physics D: Applied Physics, vol. 50, no. 10, p. 105204, 2017.

[20] R. T. Waters and W. B. Stark, "Characteristics of the stabilized glow discharge in air," Journal of Physics D: Applied Physics, vol. 8, no. 4, p. 416, 1975.

[21] W. Hermstein, "Die stromfaden-entladung und ihr übergang in das glimmen,” Archiv für Elektrotechnik, vol. 45, no. 3, pp. 209224, 1960.

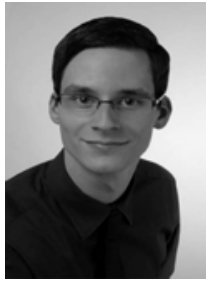

Tobias Dezenzo (S'16) was born in Offenbach am Main, Germany in 1987. He received the B. Eng. degree from the University of Applied Sciences Darmstadt, Germany in 2012, the M.Sc. degree from University of Applied Sciences Darmstadt, Germany in 2013. He is currently working toward the Ph.D. degree at Dublin Institute of Technology, Ireland. He is a teaching-fellow at University of Applied Sciences Darmstadt. His current research interests are in the field of partial discharges at dc voltage.

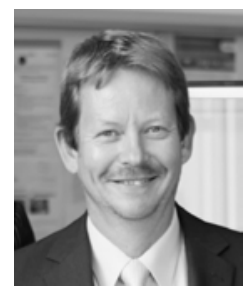

Thomas Betz received the Dr.-Ing. degree in 1997 from the Technical University of Darmstadt. $\mathrm{He}$ has 11 years industrial experience in development of high voltage gas-insulated switchgear, finally as head of development. In 2009 he joined the University of Applied Sciences in Darmstadt as professor for high voltage technology and power systems. He is member of VDE and Cigré. In this context he participated in the Cigré working group A3.27 about the impact of the application of Vacuum Switchgear at transmission voltages. The main research field is the defect prevention by partial discharge analysis and the recognition of failure causes in case of DC-applications.

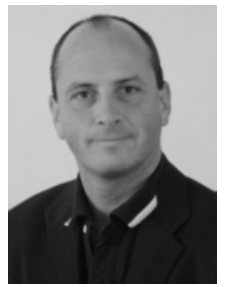

Andreas Schwarzbacher is a lecturer at the Dublin Institute of Technology, Ireland. He was awarded a degree in communications engineering by the industry financed University of Dieburg in Germany in 1995, a M.Sc. degree in computer science by the National University of Ireland, Maynooth in 2005 and a Ph.D. degree in electronics by Trinity College, University of Dublin, Ireland in 2001. Dr. Schwarzbacher has been a guest lecture in various universities and he is working for Engineers Ireland in the quality control of various degree programs. 